\title{
Optical Identification of Weak and Compact Radio Sources
}

\author{
V. S. Artyukh \\ PRAO, FIAN, 142290, Pushchino, Moscow Reg., Russia \\ M. A. Hovhannisyan, A. P. Mahtesyan, and V. H. Movsesyan \\ Byurakan Astrophysical Observatory, 378433, Byurakan, Aragatzotn \\ Province, Armenia, e-mail: martin@bao.sci.am
}

A sample of 289 compact radio sources selected from the $7 \mathrm{C}$ Catalogue, covering an area of 0.097 steradian, was surveyed at $102 \mathrm{Mhz}$ by the scintillation method. The observations show that the sizes of these sources are less than $0.1^{\prime \prime}$ and their flux densities do not exceed $2 \mathrm{Yn}$. These sources are identified with objects from the FIRST catalogue of radio sources. 99 of these objects have scintillations and have no neighbor in the surrounding $5^{\prime} .34$ of those 99 are identified with objects on the POSS within $10^{\prime \prime} \times 10^{\prime \prime}$ areas. 17 of the last 34 are closer to identified POSS objects (within $2^{\prime \prime} \times 2^{\prime \prime}$ areas). So we suppose that these 17 radio sources are clearly identified with optical sources. Most of them are probably bright quasars. The other 17 will not be discussed here, as we didn't succeed in their optical identification. There are no POSS objects brighter than $21^{m}$ in the close surroundings of the remaining 65 radio sources. We have started optical observations of areas close to those 65 objects with the $2.6-\mathrm{m}$ telescope of the Byurakan observatory. Up to now 15 areas were observed. In 5 cases optical objects were found fainter than $22^{m}-24^{m}$ within $3^{\prime \prime}$ of the compact radio sources (one example is shown in Fig. 1). We are sure that there are no optical objects brihgter than $25^{m}$ that may be identified with the remaining 10 objects. Those five identifications give us an opportunity to suppose that they are remote quasars. More powerful telescopes than the $2.6-\mathrm{m}$ are needed to perform photometric and spectral observations of similar objects in order to prove our suggestion.

\section{References}

Artyukh V.S., Tyulbashev S.A. 1996, Russian Astron J., 73, 661

Artyukh V.S., Tyulbashev S.A. 1998, Russian Astron J., 75, 655

Artyukh V.S., Tyulbashev S.A., Isaev E.A. 1998, Russian Astron J., 75, 323

McGilchhrist, M. M., Baldwin, J. E., \& Riley J. M., 1990, MNRAS, 246, 110 

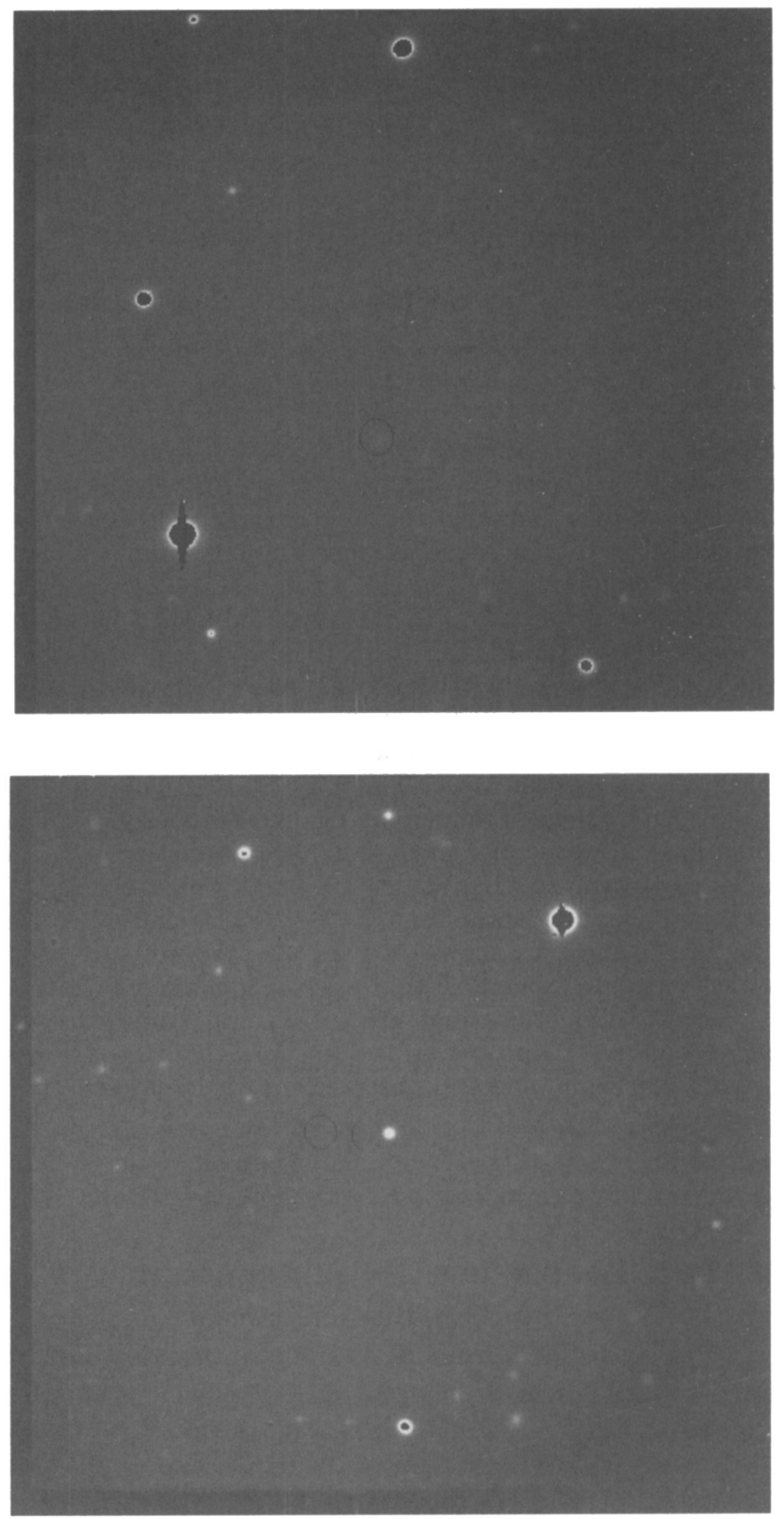

Figure 1. There are optical objects brihgter than $25^{m}$ within $3^{\prime \prime}$ of the radio sources (top) and there are no such objects in the lower field. 